УДК 512.54

\title{
Antiplane Strain of Hardening Elastoviscoplastic Medium
}

\author{
Aleksandr N. Prokudin* \\ Sergey V. Firsov ${ }^{\dagger}$ \\ Institute of Engineering and Metallurgy \\ Far East Branch of Russian Academy of Sciences \\ Metallurgov, 1, Komsomolsk-on-Amur, 681005 \\ Russia
}

Received 08.08.2017, received in revised form 20.09.2017, accepted 20.03.2018

Deforming of hardening elastoviscoplastic medium under the action of variable pressure gradient is considered in this paper. The displacement vector of material points has only vertical component. Mathematical model is based on the theory of large elastoplastic deformations. Differential transport equations of tensors of reversible and irreversible deformations are formulated. Irreversible deformations are split up into plastic and creep deformations. The solution is obtained with the use of analytical and numerical methods. The influence of hardening and viscosity parameters on medium deformation is analyzed.

Keywords: large strains, plasticity, creep, hardening.

DOI: 10.17516/1997-1397-2018-11-4-399-410.

\section{Introduction}

Appropriate theory of elastoplasticity is crucial prerequisite for correct modelling of many manufacturing and natural processes. For infinitesimal deformations such theory is wellestablished and it has become classical at the present time. Development of a theory of finite elastoplasticity involves a lot of difficulties [1,2]. In spite of significant interest of researchers and large amount of published results, there is not generally accepted theory of finite elastoplasticity so far.

One of approaches to model finite elastoplastic deformations was suggested [3,4]. In this theory tensors of reversible and irreversible deformations are introduced as thermodynamic parameters. Transport equations for these tensors are stated according to non-equilibrium thermodynamics formalism. The assumption of invariance of reversible deformations in the regions of plastic flow and invariance of irreversible deformations under unloading are also used to simplify basic equations of the theory. It should be noted that equations of this theory are reduced to classical Prandtl-Reuss equations in the case of infinitesimal deformations.

Previously a number of model initial-boundary value problems for the theory were solved analytically [5-8]. Furthermore the theory has been generalized for non-isothermal and viscous deformations $[9,10]$. Detailed derivation of the equations of the theory and the main results were presented [1]. In this paper we study deformation of incompressible material in the cylindrical tube under the action of variable pressure gradient with the use of the theory of large elastoplastic deformations $[3,4]$. No-slip boundary conditions on tube walls are adopted . We consider

\footnotetext{
*prokudin@imim.ru

†firsov.s.new@yandex.ru

(c) Siberian Federal University. All rights reserved
} 
elastoviscoplastic continuum. Such problem formulation is similar to anti-plane shear because the displacement vector has only vertical component. Anti-plane strain problem is solved for many material models [11-15]. Present work continues studies $[8,16]$ in which anti-plane strain problem was solved for elastoplastic and viscoelastic materials with the use of the theory $[3,4]$.

\section{Mathematical formulation}

Let us start with basic equations of used theory of finite elastoplasticity. We use Cartesian coordinate system and index notation. Euler coordinates are used to describe motion of continuum. Almansi strain tensor $d_{i j}$ has the form

$$
d_{i j}=\frac{1}{2}\left(u_{i, j}+u_{j, i}-u_{i, k} u_{k, j}\right),
$$

where $u_{i}$ are components of displacement vector.

Strain rate tensor and vorticity tensor have the following components:

$$
\varepsilon_{i j}=\frac{1}{2}\left(v_{i, j}+v_{j, i}\right), \omega_{i j}=\frac{1}{2}\left(v_{i, j}-v_{j, i}\right),
$$

where $v_{i}$ are components of velocity.

Deformations of continuum are split up into reversible $e_{i j}$ and irreversible $p_{i j}$ parts. We suppose that components of deformation are thermodynamic parameters along with temperature and entropy. According to $[3,4]$ we write differential transport equations of reversible and irreversible deformations as follows

$$
\begin{aligned}
\frac{D e_{i j}}{D t} & =\varepsilon_{i j}-\gamma_{i j}-\frac{1}{2}\left(\left(\varepsilon_{i k}-\gamma_{i k}+z_{i k}\right) e_{k j}-e_{i k}\left(\varepsilon_{k j}-\gamma_{k j}-z_{k j}\right)\right) \\
\frac{D p_{i j}}{D t} & =\gamma_{i j}-p_{i k} \gamma_{k j}-\gamma_{i k} p_{k j}
\end{aligned}
$$

here $\gamma_{i j}$ is a source of irreversible deformations.

Objective derivative in equations (3) is defined through total derivative and rotation tensor $r_{i j}$ :

$$
\frac{D n_{i j}}{D t}=\frac{d n_{i j}}{d t}-r_{i k} n_{k j}+n_{i k} r_{k j}, \quad \frac{d n_{i j}}{d t}=\frac{\partial n_{i j}}{\partial t}+n_{i j, j} v_{j},
$$

where $n_{i j}$ are components of arbitrary tensor.

In turn rotation tensor $r_{i j}$ consists of linear $\omega_{i j}$ part and non-linear part $z_{i j}$ :

$$
\begin{aligned}
& r_{i j}=\omega_{i j}+z_{i j}\left(e_{i j}, \varepsilon_{i j}\right), \\
& z_{i j}=-z_{i j}=A^{-1}\left(B^{2}\left(\varepsilon_{i k} e_{k j}-e_{i k} \varepsilon_{k j}\right)+\right. \\
& \left.+B\left(\varepsilon_{i k} \varepsilon_{k s} e_{s j}-e_{i k} e_{k s} \varepsilon_{s j}\right)+e_{i k} \varepsilon_{k s} e_{s t} e_{t j}-e_{i k} e_{k s} \varepsilon_{s t} e_{t j}\right), \\
& A=8-8 E_{1}+3 E_{1}^{2}-E_{2}-\frac{1}{3} E_{1}^{3}+\frac{1}{3} E_{3}, \\
& B=2-E_{1}, \quad E_{1}=e_{j j}, \quad E_{2}=e_{j m} e_{m j}, \quad E_{3}=e_{j m} e_{m n} e_{n j},
\end{aligned}
$$

where $E_{i}$ are invariants of tensor of reversible deformations.

It should be noted that if we assume that non-linear part $z_{i j}$ of rotation tensor is equal to zero then derivative (4) reduces to Jaumann derivative. 
Decomposition of total deformation $d_{i j}$ into reversible part $e_{i j}$ and irreversible part $p_{i j}$ follows from transport equations (3):

$$
d_{i j}=e_{i j}+p_{i j}-\frac{1}{2} e_{i k} e_{k j}-e_{i k} p_{k j}-p_{i k} e_{k j}+e_{i k} p_{k m} e_{m j} .
$$

Like in classical theory of infinitesimal deformations we assume that stresses in medium depend only on reversible deformations. Then we use relation analogous to the Murnaghan relation for incompressible medium [17]

$$
\sigma_{i j}=-P_{1} \delta_{i j}+\frac{\partial W}{\partial e_{i k}}\left(\delta_{k j}-e_{k j}\right), p_{i j} \neq 0,
$$

where $\sigma_{i j}$ are components of Cauchy-Euler stress tensor, $P_{1}$ is additional hydrostatic pressure, and $W$ is an elastic potential.

Relation (7) is reduced to the Murnaghan relation when irreversible deformations in continuum are absent $\left(p_{i j} \equiv 0\right)$.

Irreversible deformations are accumulated due to two different processes: creep and plastic flow. Creep deformations occur when any non-zero stresses take place and plastic deformations occurs only when stresses reach critical state. Creep rate is defined by the following law

$$
\varepsilon_{i j}^{c}=\frac{\partial V}{\partial \sigma_{i j}},
$$

where $V$ is a creep potential.

The rate of plastic deformations is defined by associated flow rule

$$
\varepsilon_{i j}^{p}=\lambda \frac{\partial f}{\partial \sigma_{i j}}, \lambda\left(\varepsilon_{i j}^{p}, p_{i j}\right)>0,
$$

where $f$ is a plastic potential.

In order to define source of irreversible deformations in transport equations we need connect rates of creep (8) and plastic flow (9). It should be noted that the source of irreversible deformations is defined differently for regions of plastic flow, viscoelastic deformation and plastic unloading. Let $\Omega$ be the whole region and $\Omega_{p}$ be the region of plastic flow. Then $\Omega / \Omega_{p}$ is the region of viscoelastic deformation and plastic unloading. The source of irreversible deformations has the form

$$
\gamma_{i j}=\left\{\begin{array}{l}
\varepsilon_{i j}^{c}, \quad x_{i} \in \Omega / \Omega_{p}, \\
\varepsilon_{i j}^{p}+\varepsilon_{i j}^{c}, \quad x_{i} \in \Omega_{p},
\end{array}\right.
$$

where $\Omega$ is the whole region, $\Omega_{p}$ is the region of plastic flow.

We assume that deformation is sufficiently slow and inertia forces can be neglected. Body forces in continuum are also neglected. Equilibrium equations in this case have the form

$$
\sigma_{i j, j}=0 .
$$

The last equation of mathematical model is the incompressibility equation

$$
\sqrt{1-2 J_{1}+2 J_{1}^{2}-2 J_{2}-\frac{4}{3} J_{1}^{3}+4 J_{1} J_{1}-\frac{8}{3} J_{3}}=1,
$$

where $J_{1}=d_{i i}, J_{2}=d_{i k} d_{k i}, J_{3}=d_{i k} d_{k j} d_{j i}$ are invariants of the Almansi tensor. 
Presented mathematical model describes large viscoelastoplastic deformations of continuum in Cartesian coordinate system in quasi-static approximation. Potentials $W, V, f$ in equations (7-9) should be chosen to specify physical properties of material. For this purpose any known potentials can used. More complex expression for the source of irreversible deformations can be used instead of (10).

Continuum fills a cylindrical tube of radius $\mathrm{R}$. Let us introduce cylindrical coordinate system $\rho, \varphi, z$. Continuum deforms due to applied pressure gradient

$$
\frac{\partial P_{1}(\rho, z, t)}{\partial z}=-\psi(t), \psi(0)=0 .
$$

Components of reversible and irreversible deformations are equal to zero at the initial point in time

$$
\left.e_{i j}\right|_{t=0}=\left.p_{i j}\right|_{t=0}=0 .
$$

No-slip condtion is set on the tube wall:

$$
\left.\vec{u}\right|_{\rho=R}=\left.\vec{v}\right|_{\rho=R}=0
$$

We assume that unknown vectors of displacement and velocity have only one non-zero component, namely, vertical component. Due to axial symmetry we obtain

$$
u=u_{z}(\rho, t), v=v_{z}(\rho, t) .
$$

Also we admit that non-linear part $z_{i j}$ of rotation tensor $r_{i j}$ is equal to zero, i.e. $r_{i j}=w_{i j}$. Non-zero components of the Almansi tensor, rate tensor and rotation tensor are

$$
\begin{gathered}
d_{\rho \rho}=-\frac{1}{2}\left(\frac{\partial u}{\partial \rho}\right)^{2}, d_{\rho z}=d_{z \rho}=\frac{1}{2} \frac{\partial u}{\partial \rho}, \\
\varepsilon_{\rho z}=\varepsilon_{z \rho}=\frac{1}{2} \frac{\partial v}{\partial \rho}, \quad w_{z \rho}=-w_{\rho z}=\frac{1}{2} \frac{\partial v}{\partial \rho} .
\end{gathered}
$$

One should note that the validity of equation of incompressibility (12) follows from equation (16). We choose Helmholtz energy as thermodynamic potential in equation (7). Assuming that density of distribution of free energy doesn't depend on irreversible deformations, we obtain $W=\rho_{0} \psi$. Then elastic potential for incompressible continuum can be written in the form of a Taylor series at a free state $\left(e_{i j}=0\right)$ :

$$
\begin{gathered}
W=W\left(I_{1}, I_{2}\right)=(\alpha-\mu) I_{1}+\alpha I_{2}+\beta I_{1}^{2}-\xi I_{1} I_{2}-\zeta I_{1}^{3}+\ldots \\
J_{1}=s_{j j}, \quad J_{2}=s_{i j} s_{j i}, s_{i j}=e_{i j}-\frac{1}{2} e_{i k} e_{k j}
\end{gathered}
$$

here $\mu, \alpha, \beta, \xi, \chi$ are material parameters.

Further we substitute the last expression in (7) and kept only terms with diagonal components up to first order and terms with non-diagonal components up to second order. This simplification is reasonable because reversible deformations are usually infinitesimal for most of structural materials. Relation for stresses have the form

$$
\begin{aligned}
& \sigma_{\rho \rho}=-\left(P_{1}+2 \mu\right)+2 b\left(e_{\rho \rho}+e_{z z}+e_{\varphi \varphi}\right)+2 \mu e_{\rho \rho}+\mu e_{\rho z}^{2}, \\
& \sigma_{\varphi \varphi}=-\left(P_{1}+2 \mu\right)+2 b\left(e_{\rho \rho}+e_{z z}+e_{\varphi \varphi}\right)+2 \mu e_{\varphi \varphi}-2 \mu e_{\rho z}^{2}, \\
& \sigma_{z z}=-\left(P_{1}+2 \mu\right)+2 b\left(e_{\rho \rho}+e_{z z}+e_{\varphi \varphi}\right)+2 \mu e_{z z}+\mu e_{\rho z}^{2}, \\
& \sigma_{\rho z}=2 \mu e_{\rho z} .
\end{aligned}
$$


Equilibrium equations (11) in cylindrical coordinates with axial symmetry (without mass and inertial forces) are

$$
\begin{aligned}
& \frac{\partial \sigma_{\rho \rho}}{\partial \rho}+\frac{\partial \sigma_{\rho z}}{\partial z}+\frac{\sigma_{\rho \rho}-\sigma_{\varphi \varphi}}{\rho}=0, \\
& \frac{\partial \sigma_{\rho z}}{\partial \rho}+\frac{\partial \sigma_{z z}}{\partial z}+\frac{\sigma_{\rho z}}{\rho}=0 .
\end{aligned}
$$

Transport equations for components of tensor of irreversible deformations are

$$
\begin{aligned}
& \gamma_{\rho \rho}=\frac{d p_{\rho \rho}}{d t}+2\left(p_{\rho \rho} \gamma_{\rho \rho}+p_{\rho z}\left(r_{z \rho}+\gamma_{\rho z}\right)\right), \\
& \gamma_{z z}=\frac{d p_{z z}}{d t}+2\left(p_{z z} \gamma_{z z}+p_{\rho z}\left(r_{\rho z}+\gamma_{\rho z}\right)\right), \\
& \gamma_{\rho z}=\frac{d p_{\rho z}}{d t}+r_{z \rho}\left(p_{\rho \rho}-p_{z z}\right)+\left(p_{\rho z}\left(\gamma_{z z}+\gamma_{\rho \rho}\right)+\gamma_{\rho z}\left(p_{z z}+p_{\rho \rho}\right)\right), \\
& \gamma_{\varphi \varphi}=\frac{d p_{\varphi \varphi}}{d t}+2 p_{\varphi \varphi} \gamma_{\varphi \varphi} .
\end{aligned}
$$

Transport equations for components of tensor of reversible deformations are

$$
\begin{aligned}
-\gamma_{\rho \rho} & =\frac{d e_{\rho \rho}}{d t}+2 r_{z \rho} e_{\rho z}, \\
-\gamma_{z z} & =\frac{d e_{z z}}{d t}+2 r_{\rho z} e_{\rho z}, \\
\varepsilon_{\rho z}-\gamma_{\rho z} & =\frac{d e_{\rho z}}{d t}+r_{\rho z}\left(e_{\rho \rho}-e_{z z}\right)+\frac{1}{2}\left(e_{\rho \rho}\left(\gamma_{\rho z}-\varepsilon_{\rho z}\right)+e_{z z}\left(\varepsilon_{\rho z}-\gamma_{\rho z}\right)+e_{\rho z}\left(\gamma_{z z}-\gamma_{\rho \rho}\right)\right), \\
-\gamma_{\varphi \varphi} & =\frac{d e_{\varphi \varphi}}{d t} .
\end{aligned}
$$

Let us define the source of irreversible deformations. We choose creep potential in equation (8) according to Norton creep law [18]

$$
\begin{aligned}
V\left(\sigma_{i j}\right) & =B \Sigma^{n}\left(\sigma_{1}, \sigma_{2}, \sigma_{3}\right), \\
\Sigma & =\max \left|\sigma_{i}-\sigma_{j}\right|,
\end{aligned}
$$

where $\sigma_{i}$ are principal components of stress tensor, $B, n$ are experimental creep parameters.

Function $\Sigma$ is defined in terms of stresses as follows

$$
\Sigma=\sqrt{4 \sigma_{\rho z}^{2}+\left(\sigma_{\rho \rho}-\sigma_{z z}\right)^{2}}
$$

Expression for the source of creep deformations follows from (8) and (24):

$$
\begin{gathered}
\varepsilon_{\rho z}^{c}=2 B n \sigma_{\rho z}\left(4 \sigma_{\rho z}^{2}+\left(\sigma_{\rho \rho}-\sigma_{z z}\right)^{2}\right)^{\frac{n}{2}-1} \\
\varepsilon_{\rho \rho}^{c}=-\varepsilon_{z z}^{c}=B n\left(4 \sigma_{\rho z}^{2}+\left(\sigma_{\rho \rho}-\sigma_{z z}\right)^{2}\right)^{\frac{n}{2}-1}\left(\sigma_{\rho \rho}-\sigma_{z z}\right) .
\end{gathered}
$$

Using the fact that $\left(\sigma_{\rho \rho}-\sigma_{z z}\right)^{2}$ is less than $\sigma_{\rho z}^{2}$, we simplify the expression for $\varepsilon_{\rho z}^{c}$ and obtain

$$
\varepsilon_{\rho z}^{c}=2^{n-1} B n \frac{\left|\sigma_{\rho z}\right|^{n}}{\sigma_{\rho z}} .
$$

It will be shown later that $\sigma_{\rho z}<0$ and hence

$$
\varepsilon_{\rho z}^{c}=(-1)^{n} 2^{n-1} B n \sigma_{\rho z}^{n-1}=(-1)^{n} 2^{n} B n \mu^{n-1} e_{\rho z}^{n-1} .
$$


Using (19), we transform relations for $\varepsilon_{\rho \rho}^{c}, \varepsilon_{z z}^{c}$ and obtain

$$
\varepsilon_{\rho \rho}^{c}=-\varepsilon_{z z}^{c}=\frac{\varepsilon_{\rho z}^{c}}{2}\left(\frac{e_{\rho \rho}-e_{z z}}{e_{\rho z}}\right) .
$$

Let us consider modification of von Mises plastic potential that includes effects of hardening and viscosity:

$$
f\left(\tau_{i j}, p_{i j}, \varepsilon_{i j}^{p}\right)=\left(\tau_{i j}-c p_{i j}-\eta \varepsilon_{i j}^{\prime p}\right)\left(\tau_{j i}-c p_{j i}-\eta \varepsilon^{\prime p}{ }_{i j}\right)-\frac{8}{3} k^{2}
$$

where $\tau_{i j}=\sigma_{i j}-\frac{1}{3} \sigma_{k k} \delta_{i j}, \varepsilon^{\prime p}{ }_{i j}=\varepsilon_{i j}^{p}-\frac{1}{3} \varepsilon_{k k}^{p} \delta_{i j}, c$ is the material parameter that accounts for the Bauchinger effect, $\eta$ is the coefficient of viscosity of plastic flow and $k$ is the yield stress.

From associated flow rule we obtain

$$
\varepsilon_{i j}^{p}=2 \lambda\left(\tau_{i j}-c p_{i j}-\eta \varepsilon_{i j}^{p}\right)
$$

Then we have

$$
\varepsilon_{i j}^{p}=\frac{2 \lambda\left(\tau_{i j}-c p_{i j}\right)}{1+2 \lambda \eta}
$$

Substituting the last formula into the relation for plastic potential $f\left(\tau_{i j}, p_{i j}, \varepsilon_{i j}^{p}\right)$, we obtain unknown function $\lambda$ :

$$
\lambda=\frac{1}{2 \eta}\left(\frac{\sqrt{\frac{3}{2}\left(\tau_{i j}-c p_{i j}\right)\left(\tau_{j i}-c p_{j i}\right)}}{2 k}-1\right)
$$

Next we get relations for the rate of plastic deformations:

$$
\begin{aligned}
\varepsilon_{i j}^{p} & =\frac{1}{\eta} \frac{Q-\sqrt{\frac{8}{3}} k}{Q}\left(\tau_{i j}-c p_{i j}\right), \\
Q & =\sqrt{\left(\tau_{i j}-c p_{i j}\right)\left(\tau_{j i}-c p_{j i}\right)} .
\end{aligned}
$$

Rates of plastic deformations can be expressed in terms of reversible deformations (19). Final relations for rates of plastic deformations have the form

$$
\begin{aligned}
& \varepsilon_{\rho \rho}^{p}=\frac{1}{3 \eta} \frac{Q-\sqrt{\frac{8}{3}} k}{Q}\left(\mu\left(4 e_{\rho \rho}-2 e_{\varphi \varphi}-2 e_{z z}+3 e_{\rho z}^{2}\right)-3 c p_{\rho \rho}\right), \\
& \varepsilon_{f f}^{p}=\frac{1}{3 \eta} \frac{Q-\sqrt{\frac{8}{3}} k}{Q}\left(\mu\left(4 e_{\varphi \varphi}-2 e_{\rho \rho}-2 e_{z z}-6 e_{\rho z}^{2}\right)-3 c p_{\varphi \varphi}\right), \\
& \varepsilon_{z z}^{p}=\frac{1}{3 \eta} \frac{Q-\sqrt{\frac{8}{3}} k}{Q}\left(\mu\left(4 e_{z z}-2 e_{\rho \rho}-2 e_{\varphi \varphi}+3 e_{\rho z}^{2}\right)-3 c p_{z z}\right), \\
& \varepsilon_{r z}^{p}=\frac{1}{\eta} \frac{Q-\sqrt{\frac{8}{3}} k}{Q}\left(2 \mu e_{r z}-c p_{\rho z}\right) .
\end{aligned}
$$


Variable Q can be defined as follows:

$$
\begin{aligned}
& Q=\sqrt{\frac{1}{3}\left(3 c^{2} B_{1}+2 c \mu B_{2}+2 \mu^{2} B_{3}\right)}, \\
& B_{1}=p_{\rho \rho}^{2}+p_{\varphi \varphi}^{2}+p_{z z}^{2}+2 p_{\rho z}^{2} \text {, } \\
& B_{2}=3 e_{\rho z}^{2}\left(-p_{\rho \rho}+2 p_{\varphi \varphi}-p_{z z}\right)+2 e_{r r}\left(-2 p_{\rho \rho}+p_{\varphi \varphi}+p_{z z}\right)+ \\
& +2 e_{\varphi \varphi}\left(p_{\rho \rho}-2 p_{\varphi \varphi}+p_{z z}\right)+2 e_{z z}\left(p_{\rho \rho}+p_{\varphi \varphi}-2 p_{z z}\right)-12 e_{\rho z}^{2} p_{\rho z} \text {, } \\
& B_{3}=12 e_{\rho z}^{2}+6 e_{\rho z}^{2}\left(e_{r r}-2 e_{f f}+e_{z z}\right)+4 e_{\rho \rho}^{2}+ \\
& +4 e_{\varphi \varphi}^{2}+4 e_{z z}^{2}-4 e_{r r} e_{z z}-4 e_{r r} e_{\varphi \varphi}-4 e_{r r} e_{z z}+9 e_{\rho z}^{4} \text {. }
\end{aligned}
$$

\section{Exact and numerical solutions}

Let us consider the second equation of equilibrium (20). Since functions $e_{\rho \rho}, e_{z z}, e_{\rho z}$ don't depend on z-coordinate then

$$
\frac{\partial \sigma_{z z}}{\partial z}=-\frac{\partial P_{1}(\rho, z, t)}{\partial z}
$$

By combining the last equation and (13) and integrating, we obtain

$$
P_{1}(\rho, z, t)=-\psi(t) z+g(\rho, t),
$$

where $g(\rho, t)$ is unknown function.

Since stress $\sigma_{\rho z}$ doesn't depend on z-coordinate the second equilibrium equation can be transformed into the following form

$$
\frac{\partial \sigma_{\rho z}}{\partial \rho}+\frac{\sigma_{\rho z}}{\rho}=-\psi(t) .
$$

General solution of this equation can written as follows

$$
\sigma_{\rho z}(\rho, t)=-\frac{\psi(t)}{2} \rho+\frac{c_{1}(t)}{\rho} .
$$

The stress $\sigma_{\rho z}$ should be finite at $\rho=0$, so $c_{1}(t)=0$ and then

$$
\sigma_{\rho z}(\rho, t)=-\frac{\psi(t)}{2} \rho
$$

Combining the last expression and (19), we obtain

$$
e_{\rho z}(\rho, t)=-\frac{\psi(t)}{4 \mu} \rho
$$

Taking into account (19), the first equilibrium equation (20) can be transformed into the following form and used for defining unknown function $g(\rho, t)$ :

$$
\frac{\partial g}{\partial \rho}+2 b\left(\frac{\partial e_{\rho \rho}}{\partial \rho}+\frac{\partial e_{z z}}{\partial \rho}+\frac{\partial e_{\varphi \varphi}}{\partial \rho}\right)+2 \mu \frac{\partial e_{\rho \rho}}{\partial \rho}+2 \mu e_{\rho z} \frac{\partial e_{\rho z}}{\partial \rho}+\rho^{-1} \mu\left(2 e_{\rho \rho}-2 e_{\varphi \varphi}+3 e_{\rho z}\right)=0
$$

Additional boundary condition is needed for defining $g(\rho, t)$ :

$$
\left.g(r, t)\right|_{r=R}=g_{0}(t)
$$


where $g_{0}(t)$ is known function of control pressure on tube wall $r=R$ at $z=0$. It was assumed that $g_{0}(t)=0$.

For convenience we introduce the following dimensionless variables

$$
\hat{\rho}=\frac{\rho}{R}, \quad \hat{z}=\frac{z}{R}, \quad \hat{u}=\frac{u}{R}, \quad \tau=\frac{t}{R} \sqrt{\frac{\mu}{\rho_{0}}}, \quad \hat{\sigma}_{i j}=\frac{\sigma_{i j}}{\mu}, \quad \hat{b}=\frac{b}{\mu}, \quad \hat{k}=\frac{k}{\mu}, \quad \hat{c}=\frac{c}{\mu},
$$

here $\rho_{0}$ is density.

Suppose that pressure gradient variation consists of three stages:

- loading, i.e. the gradient increases from 0 to maximum value $\psi_{\max }$;

- gradient is constant;

- unloading, i.e. the gradient decreases to zero.

To describe the pressure gradient we use following piecewise function

$$
\psi(\tau)=\left\{\begin{array}{l}
\frac{\psi_{\max }}{2} \cdot\left(1+\sin \left(\frac{\pi}{t_{1}} \tau-\frac{\pi}{2}\right)\right), \quad 0 \leqslant \tau \leqslant \tau_{1}, \\
\psi_{\max }, \quad \tau_{1}<\tau \leqslant \tau_{2}, \\
\frac{\psi_{\max }}{2} \cdot\left(1+\sin \left(\frac{\pi}{\left(t_{3}-t_{2}\right)}\left(\tau-\tau_{2}\right)+\frac{\pi}{2}\right)\right), \quad \tau_{2}<\tau \leqslant \tau_{3} .
\end{array}\right.
$$

We obtain system of differential equations $(21),(22),(42)$ with respect to dimensionless variables (44) with initial and boundary conditions (14), (15), (43). This system is solved numerically with the use of finite difference method. Central difference is used to approximate space derivatives and explicit scheme is used for time derivatives. Position of boundary between the region of viscoelastic deformation and the region of plastic flow is also defined numerically by following condition:

$$
Q-\sqrt{\frac{8}{3}} k=0
$$

\section{Numerical results}

The following parameters are used in numerical solution

$$
\begin{gathered}
n=3, \quad \frac{B n R \mu^{n-1} \sqrt{\rho_{0}}}{\sqrt{\mu}}=3.5, \quad \hat{\eta}=\frac{2 \sqrt{\mu} R \sqrt{\rho_{0}}}{\eta}=1.0, \quad \hat{b}=4, \quad \hat{k}=0.00125, \quad \hat{c}=0.05, \\
\tau_{1}=16, \quad \tau_{2}=32, \quad \tau_{3}=48, \quad \psi_{\max }=5 \cdot 10^{-3} .
\end{gathered}
$$

Let us consider results obtained with values (47). Fig. 1 shows the variation of elasto-plastic boundary in time. Position of boundary $\hat{\rho}=1$ is used in the case when plastic flow doesn't occur in the whole region. Yield criterion firstly holds on the cylinder wall at instant $\tau \cong 8.8$, after that elastoplastic boundary moves towards the centre of the cylinder. At instant $\tau_{1}$ region of plastic flow occupies more than $40 \%$. One can see when load is constant during interval from $\tau_{1}$ to $\tau_{2}$, the boundary slowly moves back to cylinder wall due to hardening of material. After instant $\tau_{2}$ the pressure gradient begins to decrease and the region of plastic flow in cylinder is reduced rapidly, and at time $\tau \cong 35.8$ it completely vanishes. 


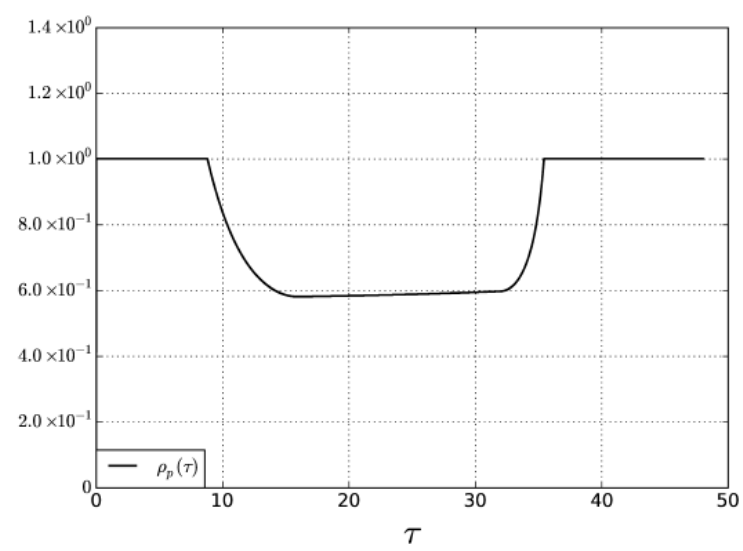

Fig. 1. Variation of elastoplastic boundary in time

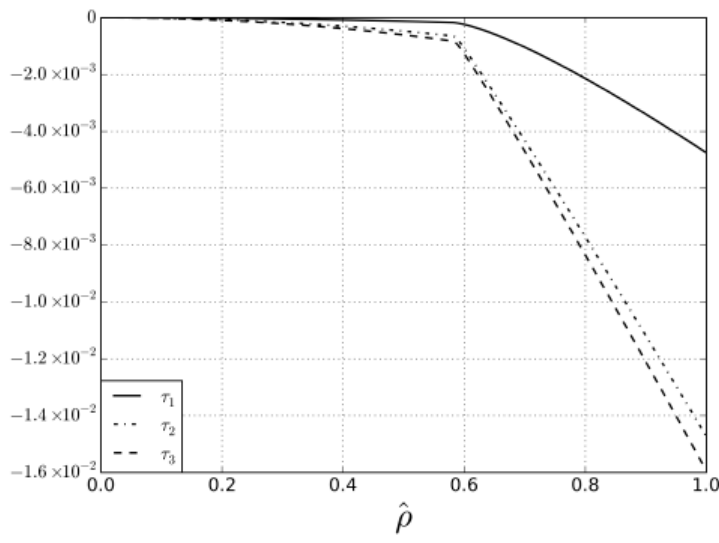

(a) Irreversible deformation $p_{r z}$

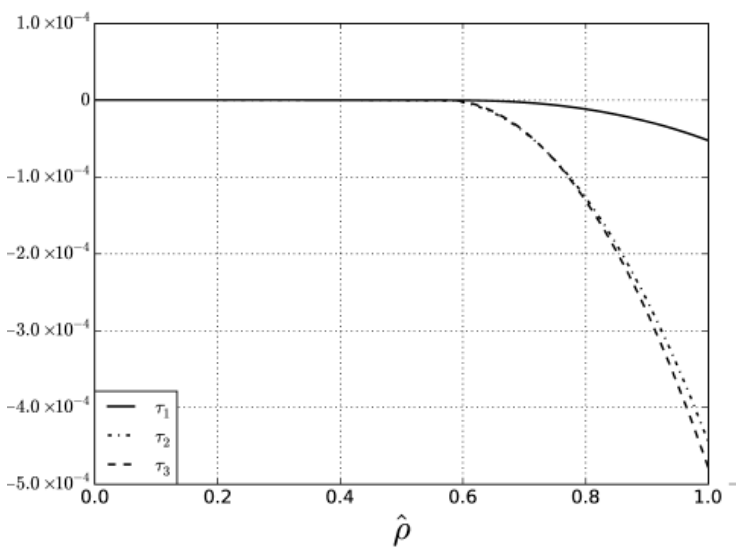

(c) Irreversible deformation $p_{r r}$

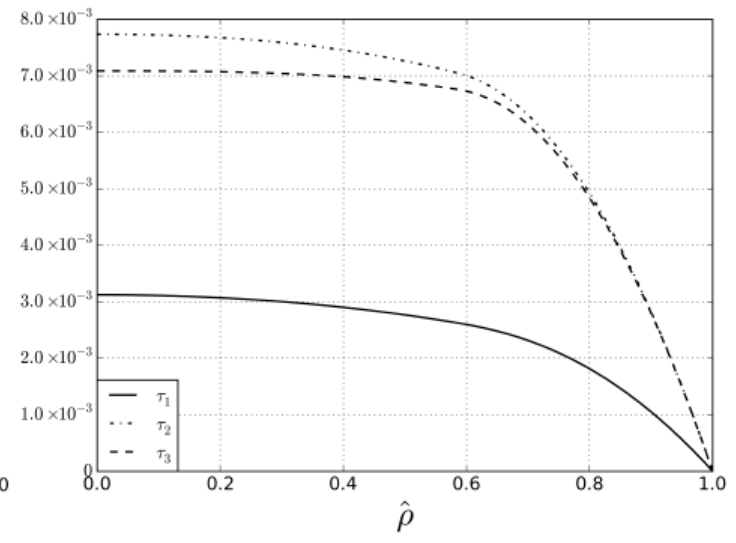

(b) Displacement $u$

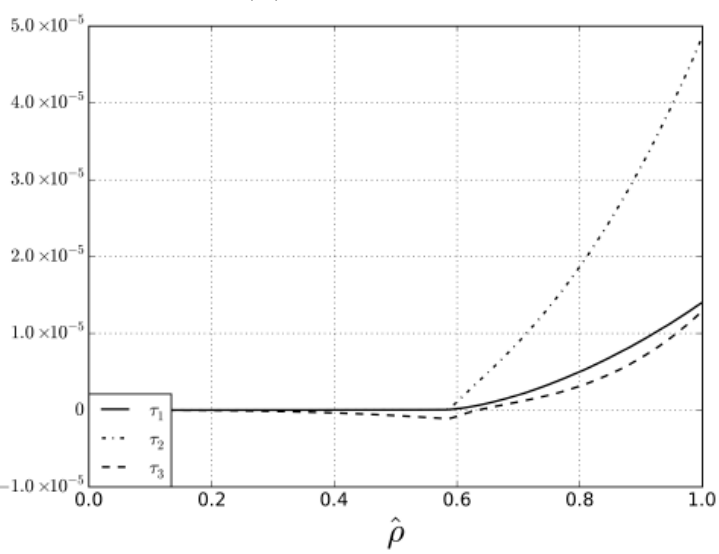

(d) Irreversible deformation $p_{z z}$

Fig. 2. Distributions of deformations and displacement at instants $\tau_{1}, \tau_{2}, \tau_{3}$

Distributions of irreversible deformations $p_{\rho z}, p_{\rho \rho}, p_{z z}$ and displacement $u$ for instants $\tau_{1}, \tau_{2}, \tau_{3}$ are shown in Fig. 2. We can see that non-diagonal component $p_{\rho z}$ of irreversible defor- 
mations is two or three orders of magnitude larger than diagonal components. Also irreversible deformations (all components) are larger in the region where plastic flow takes place. In the interval from $\tau_{1}$ to $\tau_{2}$ when the pressure gradient is decreased level of irreversible deformation $p_{z z}$ is also decreased.Deformation $p_{\rho z}, p_{\rho \rho}$ are slightly increased. Reversible deformations $e_{\rho \rho}, e_{z z}$ vary in a similar way.

Next we examine the influence of hardening parameter on variation of elastoplastic boundary and on accumulation of irreversible deformations in the cylinder. To do this we carry out series of computations with parameters (47) but for three values of $\hat{c}: \hat{c}=0,0.05,0.25$. Variations of elastoplastic boundary in time are shown in Fig. 3. When hardening doesn't occur $(\hat{c}=0)$, plastic flow has the most duration and extends to the largest part of the cylinder. Increasing parameter $\hat{c}$ leads to the decrease of plastic flow duration. Fig. 4 shows distributions of irreversible deformation $p_{\rho z}$ and displacement $\hat{u}$ at instant $\tau_{3}$, i.e. residual deformation and displacement. As we can see from these figures increasing parameter $\hat{c}$ leads to the decrease of irreversible deformation and displacement in the cylinder.

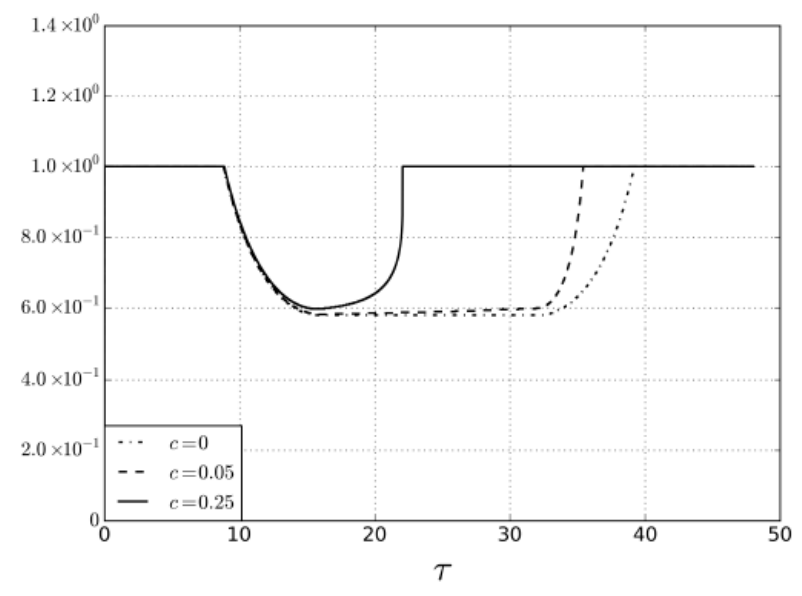

Fig. 3. Variation of elastoplastic boundary in time

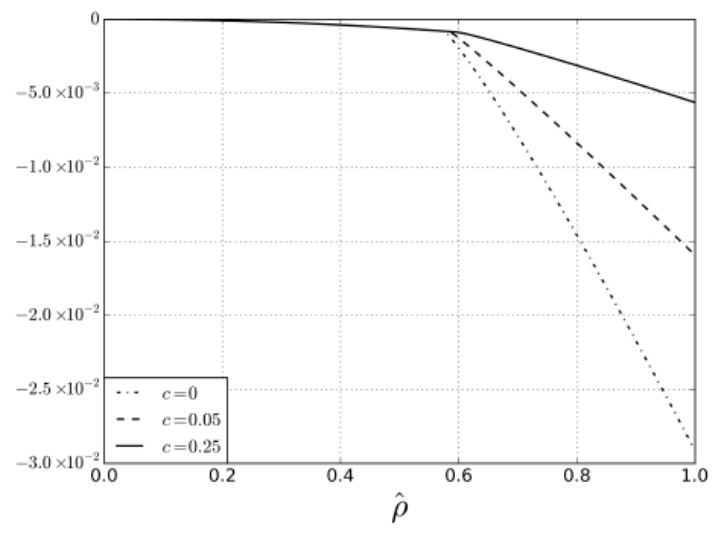

(a) Irreversible deformation $p_{r z}$

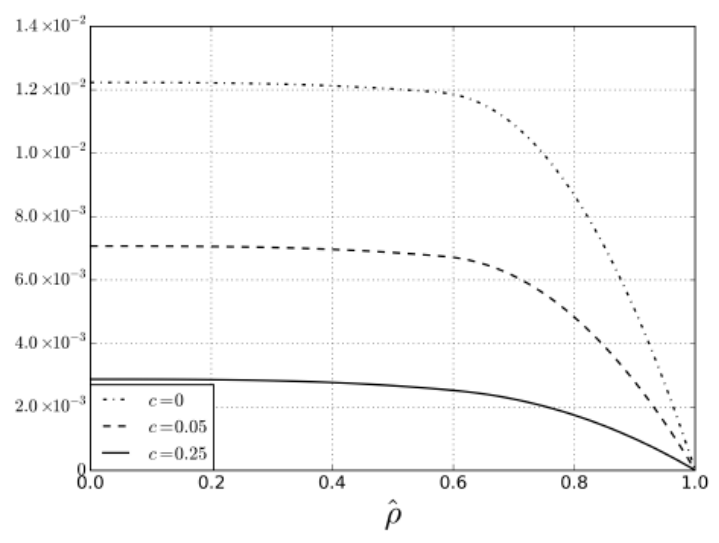

(b) Displacement $u$

Fig. 4. Numerical results for $\hat{c}=0,0.05,0.25$ at time $\tau_{3}$ 
Finally we examine the influence of material viscosity (parameter $\hat{\eta}$ ) on plastic flow. Computations for $(47)$ and $\hat{\eta}=0.2,1.0,5.0$ were carried out. Numerical results show that viscosity has no effect on position of elastoplastic boundary between 0 and $\tau_{1}$ (the pressure gradient is increased). Also it was established that the increase of viscosity leads to deceleration of plastic flow and the decrease of residual plastic deformations. And vice versa, duration of plastic flow is decreased but there are more considerable accumulation of deformations when viscosity is decreased.

\section{References}

[1] A.A.Burenin, L.V.Kovtanyuk, Large irreversible strains end elastic aftereffect, Vladivostok, Dal'nauka, 2013 (in Russian).

[2] X.Heng, O.T.Bruhns, A.Meyers, Elastoplasticity beyond small deformations, Acta mechanica, 182(2006), 31-111.

[3] A.A.Burenin, G.I.Bykovtsev, L.V.Kovtanyuk, A simple model of finite strain in an elastoplastic medium, Doklady Physics, 41(1996), no. 3, 127-129.

[4] V.P.Myasnikov, Equations of motion of elastoplastic materials under large strains, Vestnik DVO RAN, (1996), no. 4, 8-13.

[5] A.A.Burenin, L.V.Kovtanyuk, M.V.Polonik, The formation of a one-dimensional residual stress field in the neighbourhood of a cylindrical defect in the continuity of an elastoplastic medium, Journal of Applied Mathematics and Mechanics, 67(2003), no. 2, 283-292.

[6] A.A.Burenin, L.V.Kovtanyuk, A.L.Mazelis, The pressing of an elastoviscoplastic material between rigid coaxial cylindrical surfaces, Journal of Applied Mathematics and Mechanics, 70(2006), no. 3, 437-445.

[7] A.A.Burenin, L.V.Kovtanyuk, On elastic strains and a viscoplastic flow in a heavy layer placed on an inclined plane, Mechanics of Solids, 45(2010), no. 2, 284-294.

[8] A.A.Burenin, L.V.Kovtanyuk, The development and deceleration of the flow of an elastoplastic medium in a cylindrical tube, Journal of Applied Mathematics and Mechanics, 77(2013), no. $5,566-572$.

[9] A.A.Burenin, L.V.Kovtanyuk, G.L.Panchenko, Modeling of large elastoviscoplastic deformations with thermophysical effects taken into account, Mechanics of Solids, 45(2010), no. 4, $583-594$.

[10] A.A.Burenin, L.V.Kovtanyuk, A.L.Mazelis, Development of a rectilinear axisymmetric viscoplastic flow and elastic aftereffect after its stop, Journal of Applied Mechanics and Technical Physics, 51(2010), no. 2, 261-268.

[11] Yu.N.Rabotnov, Deformable solid mechanics, Moscow, Nauka, 1988 (in Russian).

[12] G.P.Cherepanov, An elastic-plastic problem under conditions of antiplanar deformation, Journal of Applied Mathematics and Mechanics, 26(1962), no. 4, 697-708. 
[13] B.D.Annin, V.D.Bondar, Antiplane strain in a nonlinearly elastic incompressible body, Journal of Applied Mechanics and Technical Physics, 47(2006), no. 6, 849-856.

[14] V.D.Bondar, Antiplane strain of a body undergoing large-rotations, Journal of Applied Mechanics and Technical Physics, 48(2007), no. 3, 460-466.

[15] V.D.Bondar, Elastoplastic antiplane strain in an incompressible body, Journal of Applied Mechanics and Technical Physics, 55(2014), no. 1, 19-29.

[16] S.V.Firsov, A.N.Prokudin, Antiplane axisymmetric creep deformation of incompressible medium, Journal of Siberian Federal University. Mathematics and Physics, 8(2015), no. 4, $406-415$.

[17] A.I.Lurie, Theory of Elasticity, Springer, Berlin, 2005.

[18] F.N.Norton, The creep steel of high temperature, NY, McGraw Hill, 1929.

\title{
Антиплоская деформация упрочняющейся упруговязкопластической среды
}

\section{Александр Н. Прокудин \\ Сергей В. Фирсов}

Институт машиноведения и металлургии ДВО РАН

Металлургов, 1, Комсомольск-на-Амуре, 681005

Россия

\begin{abstract}
В работе рассмотрена деформация упрочняющейся упруговязкопластической средь под действием изменяющегося перепада давления. Вектор перемещений точек среды имеет только вертикальную компоненту. Математическая модель основана на теории больших упругопластических деформаций, в рамках которой сформулированы дифференциалъные уравнения переноса тензоров обратимых и необратимых дебормаций. При этом необратимые деформации включают в себя деформачии ползучести и пластические деформачии. Решение задачи получено с исполъзованием аналитических и численных методов. Проанализировано влияние параметров упрочнения и вязкости на деформирование среды.
\end{abstract}

Ключевые слова: конечные деформации, пластичность, ползучесть, упрочнение. 